\title{
Exact Kohn-Sham potential of strongly correlated finite systems
}

\author{
N. Helbig, ${ }^{1, a)}$ I. V. Tokatly, ${ }^{1,2}$ and A. Rubio ${ }^{1,3}$ \\ ${ }^{1}$ Nano-Bio Spectroscopy Group and ETSF Scientific Development Centre, Dpto. Física de Materiales, \\ Universidad del País Vasco, Centro de Física de Materiales CSIC-UPV/EHU-MPC and DIPC, \\ Av. Tolosa 72, San Sebastián E-20018, Spain \\ ${ }^{2}$ IKERBASQUE, Basque Foundation for Science, Bilbao E-48011, Spain \\ ${ }^{3}$ Fritz-Haber-Institut, Max-Planck-Gesellschaft, Berlin D-14195, Germany
}

(Received 5 August 2009; accepted 13 November 2009; published online 9 December 2009)

\begin{abstract}
The dissociation of molecules, even the most simple hydrogen molecule, cannot be described accurately within density functional theory because none of the currently available functionals accounts for strong on-site correlation. This problem led to a discussion of properties that the local Kohn-Sham potential has to satisfy in order to correctly describe strongly correlated systems. We derive an analytic expression for the nontrivial form of the Kohn-Sham potential in between the two fragments for the dissociation of a single bond. We show that the numerical calculations for a one-dimensional two-electron model system indeed approach and reach this limit. It is shown that the functional form of the potential is universal, i.e., independent of the details of the two fragments. () 2009 American Institute of Physics. [doi:10.1063/1.3271392]
\end{abstract}

\section{INTRODUCTION}

Over the years the improvement in exchange-correlation (xc) functionals made density functional theory $(\mathrm{DFT})^{1,2}$ the tool of choice to accurately study and predict properties of many-electron systems. Applications range from atoms to molecules and nanostructures, biomolecules, and solids and cover diverse topics such as theoretical spectroscopy, e.g., optical, energy loss, and time-resolved spectroscopy, electron transport, light induced phase transitions, photochemistry, and electrochemistry. ${ }^{3,4}$ Despite this success major basic challenges remain that usually are manifestations of strong, static, and dynamic electron correlations. ${ }^{5}$ Van der Waals interactions, the localization in strongly correlated systems, open-shell molecules, and molecular dissociation are poorly accounted for by present functionals. ${ }^{5,6}$ A general measure of interelectron correlations is the ratio of the kinetic energy to the potential energy of the Coulomb interaction between electrons. While the kinetic energy is lowered by delocalization of electrons over the system, the Coulomb repulsion works in the opposite direction trying to keep electrons far from each other and thus favoring the tendency to localization. In the condensed matter context this interplay of two opposite tendencies is commonly pictured in terms of the Hubbard on-site correlations that suppress tunneling of particles between atoms and lead to localization of electrons on lattice sites (or groups of sites). Strong Hubbard correlations are responsible for the dissociation of molecules, the physics of Mott insulators, nonitinerant magnetism in most of the magnetic dielectrics, the Coulomb blockade in quantum transport, etc. The failure of the common DFT-functionals to capture the effects of Hubbard correlations led to the development of the LDA+U method ${ }^{7}$ and its more elaborated counterpart, the dynamical mean-field theory (DMFT), ${ }^{8}$ to

${ }^{a)}$ Electronic mail: nehelbig@gmail.com. describe strongly correlated systems. On the other hand, it is absolutely clear that DFT being an "in principle exact" theory should be capable to describe the regime of strong correlations provided the proper xc potential is known. In this realm, it is fundamental to increase the knowledge of relations, fulfilled by the exact xc potential, in order to move forward on the road toward the ultimate functional, the "holy grail of DFT."

In the present work, we consider a prototypical example of a physical behavior governed by strong Hubbard correlations - the dissociation of diatomic molecules, and discuss exact features of the xc potential $v_{x c}$ necessary to describe the correlation-driven electron localization happening in the dissociation limit. One such feature is well known; in the dissociation of heteroatomic molecules the KohnSham (KS) potential $v_{s}$ acquires a step in between the fragments to adjust the ionization potentials. ${ }^{9}$ The value of this step is universal and is simply given by the difference of the ionization potentials of the two fragments of the dissociated molecule. Apparently, the presence of this step is necessary to prevent an unphysical fall of electrons to the fragment with a higher ionization potential. However, as we discuss below, it is not sufficient to correctly describe the dissociation, i.e., the strongly correlated, limit. In fact, in this limit the xc potential acquires a nontrivial structure even for the most simple homoatomic molecules, such as $\mathrm{H}_{2}$.

An important step in understanding the behavior of the $\mathrm{xc}$ potential in the dissociation limit has been made in a series of works by Baerends and co-authors, ${ }^{10-15}$ who reconstructed the xc potential of a number of stretched diatomic molecules from an accurate many-body configuration interaction (CI) ground-state wave function. They noticed that, in addition to the step, $v_{x c}$ also shows a peak structure around the middle point between the two atoms. ${ }^{10-14}$ A subsequent analysis has shown that the peak in $v_{x c}$ is a general feature of the dissociation limit, which contradicts the common LCAO 
form of the molecular orbital, but can be reasonably well reproduced assuming that the two-electron wave function is of Heitler-London form constructed out of the atomic KS orbitals. $^{15,16}$ The one-dimensional (1D) Heitler-London model wave function was, very recently, used to discuss the height and position of the peak and step. ${ }^{16}$ By partitioning the $\mathrm{xc}$ potential Baerends and co-workers showed that the peak originates from the "kinetic correlation term." "10,14 Additionally, they interpreted the peak as a term that must balance a well in the KS kinetic energy density, and make the resulting density more localized on the atoms. ${ }^{15}$ The physical nature of the peak structure and its connection with the Hubbard correlations in the dissociation of a single bond is the main subject of our paper. We prove that the spatial dependence of the KS potential in between the fragments in the strongly correlated dissociation limit, including both the peak structure and the step (for heteroatomic molecules), is universal. ${ }^{17}$ It depends only on the asymptotic behavior of the density of the fragments, which, in turn, is mainly determined by the atomic ionization potentials. ${ }^{9}$ In particular, we derive an analytic formula that allows us to recover the exact form of the xc potential in regions far away from the two fragments in the dissociation limit from the knowledge of the ionization potentials of the independent fragments. This result adds one more item to the list of exact properties of the KS system and xc potential, such as Koopman's theorem, the exact asymptotic form of $v_{x c}$ for finite systems, and the exact relation of the asymptotics of the density to the asymptotics of the highest occupied KS state. ${ }^{9}$ We also demonstrate that the peak structure in $v_{x c}$ can be viewed as a manifestation of the Hubbard on-site correlations at the level of noninteracting KS particles. The physical significance of this peak is that it suppresses the quantum tunneling of $\mathrm{KS}$ particles between two fragments, exactly what the Hubbard repulsion does for real electrons. This ensures that the fragments become physically independent. To the best of our knowledge, only the nonlocal functionals of Becke ${ }^{18}$ and Perdew ${ }^{19}$ show a peak in the dissociation limit, however, both functionals do not yield the correct limit at infinite distance. ${ }^{20}$ Hence, further improvement of functionals is necessary, and the results presented here constitute a stringent test for the future development of static and time-dependent functionals aimed at describing strongly correlated systems.

The structure of the paper is as follows. In Sec. II we discuss the physics of the strongly correlated dissociation limit in terms of both Hubbard on-site correlations and the KS formulation of DFT. Using a simple analytically solvable model for a $1 \mathrm{D}$ symmetric diatomic we derive the asymptotic form of the KS potential and verify our findings numerically for more general symmetric 1D systems. In Sec. III we uncover the universal physics that governs the behavior of the KS potential in the dissociation limit, derive general exact analytic formulas valid for all two-electron systems, and verify them numerically for model $1 \mathrm{D}$ heteroatomic molecules. We also discuss generalizations of the results for more general many-electron systems. We then conclude the paper by summarizing our main results. An Appendix is included to connect the results presented here to the previous works by Baerends and co-workers. ${ }^{10-15,20}$

\section{TOWARD DFT IN THE DISSOCIATION LIMIT}

\section{A. Physics of the dissociation limit: Real electrons versus Kohn-Sham particles}

Let us first consider the qualitative physics of the dissociation of simple diatomic molecules with a single $\sigma$-bond formed by a pair of electrons originating from the atomic valence orbitals. Specific examples for this scenario include $\mathrm{H}_{2}, \mathrm{Li}_{2}$, and $\mathrm{LiH}$, to name a few. When the molecule is stretched, the gain in the kinetic energy due to the delocalization of the electrons, which is proportional to the hopping matrix element $t$, decreases exponentially. On the other hand, the loss in the interaction energy, due to the presence of two electrons on the same atom, saturates at a certain value of the Hubbard on-site repulsion $U$. Starting from some distance, roughly determined by the condition $U \gtrsim t$, the on-site Coulomb correlations block the interatomic tunneling, the electrons get localized on their own atoms, and the molecule dissociates into two physically independent fragments.

Within DFT the real interacting system is modeled by an artificial noninteracting KS system with the same groundstate density. The noninteracting particles are subject to an effective potential via the KS (Ref. 2) equation (atomic units are used throughout the paper),

$$
\left[-\frac{\nabla^{2}}{2}+v_{s}(\mathbf{r})\right] \varphi_{j}(\mathbf{r})=\epsilon_{j} \varphi_{j}(\mathbf{r})
$$

Since the KS particles are noninteracting there is no way to localize them on a particular atom, independent of the distance $d$ between the fragments. For a symmetric molecule, like $\mathrm{H}_{2}$ or $\mathrm{Li}_{2}$, the $\mathrm{KS}$ particles responsible for the formation of the bond always occupy a symmetric orbital with a probability of $1 / 2$ to find either particle on each atom.

Apparently, the behavior of the KS particles is very different from that of real physical electrons. The difference between the real world and an artificial world of KS particles becomes especially striking in the regime of strong correlations, and the dissociation of simple molecules provides us with a bright example of this phenomenon. However, a certain physical information, namely, the ground-state density, is reproduced exactly by the $\mathrm{KS}$ system. Therefore, the real physics should be reflected in the properties of the KS system. Establishing a map of the physics governed by the strong Hubbard on-site correlations to the properties of the KS potential, i.e., the map of the real world to the world of $\mathrm{KS}$ particles, is the main subject of this work. In order to find this map we mainly concentrate on a minimal model that captures all key physics of dissociation-the system of two electrons in a potential formed by two nuclei/potential wells. In Sec. III we argue that the main conclusions are transferable to a more general many-electron case.

In the case of two electrons in a singlet state only one spatial KS orbital is occupied. Therefore, the density is given as $n(\mathbf{r})=2\left|\varphi_{1}(\mathbf{r})\right|^{2}=2 \varphi_{1}^{2}(\mathbf{r})$, because the orbital can always be chosen to be real. Hence, from inverting Eq. (1), the exact $\mathrm{KS}$ potential is given by 


$$
v_{s}(\mathbf{r})=\frac{1}{2} \frac{\nabla^{2} \sqrt{n(\mathbf{r})}}{\sqrt{n(\mathbf{r})}}+\epsilon_{1}
$$

with $n(\mathbf{r})$ being, by construction, the exact ground-state density of the two-electron system. Hence, given the exact twobody ground-state wave function $\Psi\left(\mathbf{r}_{1}, \mathbf{r}_{2}\right)$, one can calculate the density $n(\mathbf{r})=\int d \mathbf{r}_{2}\left|\Psi\left(\mathbf{r}, \mathbf{r}_{2}\right)\right|^{2}$, and then recover the exact KS potential by inserting $n(\mathbf{r})$ into Eq. (2). This formally maps the physical two-body wave function to the KS potential. However, extracting the physics behind this formal map is not as simple as one may think since in a general threedimensional (3D) case the wave function $\Psi\left(\mathbf{r}_{1}, \mathbf{r}_{2}\right)$ is a complicated object given fully numerically, e.g., from CI calculations, and, moreover, may be numerically problematic for realistic systems when one reaches the dissociation limit. Therefore, it is instructive to look first at some simplified models and then, after the essential physics is understood, return to realistic situations.

An obvious simplification, which still contains all physical ingredients of the original problem, is to consider a system of two interacting particles in one dimension. The corresponding two-electron Schrödinger equation takes the form

$$
\begin{aligned}
& {\left[-\frac{1}{2}\left(\frac{\partial^{2}}{\partial x_{1}^{2}}+\frac{\partial^{2}}{\partial x_{2}^{2}}\right)+v_{\text {ext }}\left(x_{1}\right)+v_{\text {ext }}\left(x_{2}\right)+v_{\text {int }}\left(\left|x_{1}-x_{2}\right|\right)\right]} \\
& \quad \times \Psi\left(x_{1}, x_{2}\right)=E \Psi\left(x_{1}, x_{2}\right),
\end{aligned}
$$

where $v_{\text {ext }}(x)$ is the external potential, and $v_{\text {int }}\left(\left|x-x^{\prime}\right|\right)$ is the potential of the interparticle interaction. At the end of this section and in Sec. III we present the results based on the full numerical solution of Eq. (3). However, to gain some physical insight into the shape of $v_{s}$ in the dissociation limit, we simplify the model even further to make it analytically solvable.

\section{B. Analytical model of strongly correlated electrons}

First, we assume that the external potential in Eq. (3) is given by a sum of two $\delta$-function wells of equal strength, $v$, located at the points $x= \pm d / 2$. Similarly, we take the interaction to be a zero-range delta-potential of strength $\lambda$,

$$
\begin{aligned}
& v_{\text {ext }}(x)=-v[\delta(x-d / 2)+\delta(x+d / 2)], \\
& v_{\text {int }}\left(\left|x-x^{\prime}\right|\right)=\lambda \delta\left(x-x^{\prime}\right)
\end{aligned}
$$

Physically, in the dissociation limit the only role of the interaction is to block the interatomic tunneling. Therefore, in that limit, the behavior is expected to be universal and independent of a particular form and/or strength of the interaction. This leads us to the last simplifying assumption, namely, the limit of infinitely strong $\delta$-repulsion, $\lambda \rightarrow \infty$. Now the problem becomes immediately solvable by the so called Girardeau mapping ${ }^{21}$ (see also a more recent review), ${ }^{22}$ which allows to map the ground state of strongly interacting "hard-core" bosons (a symmetric wave function) to the ground state of noninteracting fermions (antisymmetric wave function). In our two-particle case the exact groundstate (singlet, i.e., symmetric) wave function takes the form

$$
\Psi\left(x_{1}, x_{2}\right)=\left|\phi_{1}\left(x_{1}\right) \phi_{2}\left(x_{2}\right)-\phi_{2}\left(x_{1}\right) \phi_{1}\left(x_{2}\right)\right|,
$$

where $\phi_{1}(x)$ and $\phi_{2}(x)$ are the two lowest states of the following one-particle Schrödinger equation:

$$
-\frac{1}{2} \phi_{n}^{\prime \prime}(x)+v_{\text {ext }}(x) \phi_{n}(x)=\epsilon_{n} \phi_{n}(x) .
$$

In other words, the ground state of two infinitely interacting particles in a singlet state is given by the modulus of the ground-state wave function of two noninteracting spinless fermions in the bare external potential $v_{\text {ext }}(x)$.

The two lowest energy solutions of Eq. (7) with the external potential of Eq. (4) are easily found to be $\phi_{1}(x)$ $=\phi_{+}(x)$ and $\phi_{2}(x)=\phi_{-}(x)$ with

$$
\phi_{ \pm}(x)=C_{ \pm}\left(e^{-\alpha_{ \pm}|x+d / 2|} \pm e^{-\alpha_{ \pm}|x-d / 2|}\right),
$$

where $C_{ \pm}$are the normalization constants. The parameters $\alpha_{ \pm}$, which determine the corresponding eigenvalues $\epsilon_{1,2}$ $=\epsilon_{ \pm}=-\alpha_{ \pm}^{2} / 2$, are the solutions of the following dispersion equations: ${ }^{23}$

$$
\alpha_{ \pm}=v\left(1 \pm e^{-\alpha_{ \pm} d}\right) .
$$

Using the exact ground-state wave function (6) we obtain the exact density

$$
n(x)=\int d x^{\prime} \Psi^{2}\left(x, x^{\prime}\right)=\phi_{+}^{2}(x)+\phi_{-}^{2}(x),
$$

and, finally, by inserting $n(x)$ into the 1D version of Eq. (2), the exact KS potential for our strongly correlated twoparticle system, $v_{s}(x)=\Delta v_{s}(x)+v_{\text {ext }}(x)$,

$$
\Delta v_{s}(x)=\frac{\left(\phi_{+} \phi_{-}^{\prime}-\phi_{+}^{\prime} \phi_{-}\right)^{2}}{2\left(\phi_{+}^{2}+\phi_{-}^{2}\right)^{2}}-\frac{\epsilon_{+} \phi_{+}^{2}+\epsilon_{-} \phi_{-}^{2}}{\phi_{+}^{2}+\phi_{-}^{2}}-\frac{v^{2}}{2} .
$$

Equation (11) gives the exact KS potential for any distance between the wells. In the dissociation limit, $v d \gg 1, \alpha_{ \pm} \rightarrow v$, and $\epsilon_{ \pm} \rightarrow-v^{2} / 2$. Therefore, the last two terms in Eq. (11) cancel while the remaining first term simplifies to

$$
\Delta v_{s}(x)=\frac{v^{2}}{2 \cosh ^{2}(2 v x)} \equiv \frac{I}{\cosh ^{2}(2 \sqrt{2 I} x)} .
$$

$I=v^{2} / 2$ is the ionization potential of a separate fragment, the delta-potential of strength $v$. Hence, we found that the exact $\mathrm{KS}$ potential in the dissociation limit has the form of a peak built up between the two fragments of the "molecule." The shape of this peak looks quite close to the peak structure observed in previous works. ${ }^{10-13}$ It also confirms previous observations that the height of the peak equals the ionization potential. $^{10,20}$

\section{1D model for homoatomic dissociation}

It is physically plausible to expect that the behavior in the dissociation limit is independent of the particular form and strength of the interaction, and that the asymptotic form of $v_{s}(x)$ for more general systems is similar to that given by the simple formula (12). We now verify this expectation for a 1D system of two particles in a more general, but still symmetric, external potential, namely, 


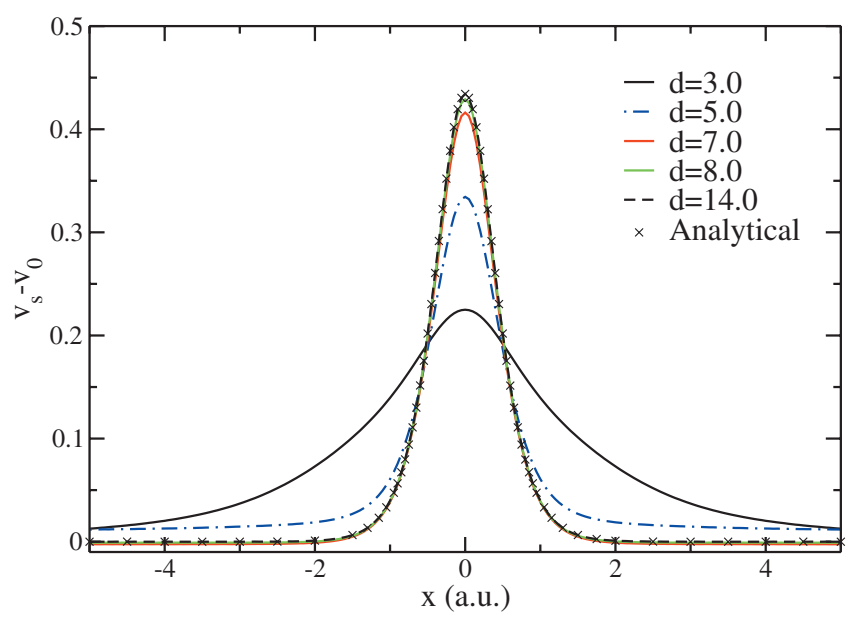

FIG. 1. KS potential for two equivalent wells at different distances $(v=0.9)$. The external potential has been subtracted to facilitate the comparison. Analytical results are given by Eq. (12).

$$
v_{\mathrm{ext}}(x)=-v\left[\frac{1}{\cosh ^{2}(x-d / 2)}+\frac{1}{\cosh ^{2}(x+d / 2)}\right] .
$$

The two particles are interacting with a finite range interaction potential of the form

$$
v_{\text {int }}(|x-y|)=\frac{b}{\cosh ^{2}(x-y)} .
$$

The choice of the $1 / \cosh ^{2}$ shape of the wells and the interaction potential is arbitrary. It is simply a matter of convenience as the 1D Schrödinger equation with a $1 / \cosh ^{2}$ potential is exactly solvable, ${ }^{24}$ which allows us to control the accuracy of our numerical calculations. In addition, the finite-range interaction (14) allows us to reach the dissociation limit in a controllable way without numerical instabilities.

For the numerical solution of Eq. (3) with general $v_{\text {ext }}$ and $v_{\text {int }}$, we note that the 1D two-particle problem defined by Eq. (3) can be formally interpreted as a 2D one-particle problem with the Hamiltonian

$$
H^{2 \mathrm{D}}=-\frac{1}{2}\left[\frac{\partial^{2}}{\partial x^{2}}+\frac{\partial^{2}}{\partial y^{2}}\right]+v_{\mathrm{ext}}^{2 \mathrm{D}}(x, y),
$$

where the effective 2D one-particle potential is defined as

$$
v_{\text {ext }}^{2 \mathrm{D}}(x, y)=v_{\text {ext }}(x)+v_{\text {ext }}(y)+v_{\text {int }}(|x-y|) .
$$

Consequently, the exact ground-state wave function $\Psi(x, y)$ and the exact 1D ground-state density for the physical twoparticle system, $n(x)=\int d y|\Psi(x, y)|^{2}$, can be obtained numerically from any computer code that is able to treat noninteracting electrons in two dimensions. All our calculations in this work were carried out with the OCTOPUS code. ${ }^{25}$

The exact KS potential, $v_{s}(x)$, for $v_{\text {ext }}$ and $v_{\text {int }}$ of Eqs. (13) and (14) with $v=0.9$ and $b=0.5$, and varying interwell distance $d$ is shown in Fig. 1. At first sight, the results look very surprising: starting from a certain distance, $d=8$ a.u. for these particular parameters, the shape of the KS potential saturates exactly at the form given by the analytic formula (12) with $I$ being the ionization potential of a single $1 / \cosh ^{2}$ well. Calculations for different strengths of the wells $v$, dif- ferent interaction strength $b$, as well as for a long-range softCoulomb interparticle interaction all show the same result. ${ }^{26}$ At large distances the exact KS potential is not only similar to the analytic form of Eq. (12), obtained from an oversimplified model with an infinite $\delta$-repulsion, but matches it $e x$ actly as soon the dissociation limit is reached! In the next section we discuss a deep, though simple physical reason for this seemingly surprising universality.

\section{EXACT KOHN-SHAM POTENTIAL IN THE DISSOCIATION LIMIT}

\section{A. Universality of the Kohn-Sham potential}

In order to understand the nature of the universal peak in the asymptotic form of the KS potential, we turn back to our first simple model with an infinite zero-range repulsion and look more closely at the behavior of the exact density determined by Eq. (10). In the dissociation limit, $v d \gg 1$, the functions $\phi_{+}(x)$ and $\phi_{-}(x)$ become simple symmetric and antisymmetric combinations of "atomic" orbitals. Taking the squares and summing them up, as suggested by Eq. (10), we find that all interference terms, i.e., the cross product of different atomic orbitals, cancel, and the total density reduces to a sum of two atomic densities,

$$
n(x)=v e^{-2 v|x-d / 2|}+v e^{-2 v|x+d / 2|} .
$$

This is exactly what Hubbard on-site correlations do-they destroy the interatomic tunneling/interference, which localizes the electrons on separate sites, and eventually makes the density to be the sum of the densities of two physically independent fragments. On the KS side of the mirror, the KS potential, whatever it is, cannot localize the KS particles. However, by building up a self-consistent peak between the fragments, it suppresses the tunneling/interference of the atomic KS orbitals to mimic the density distribution of the two independent atoms. Thus, the physics of the on-site Hubbard correlations in the real world is mapped to the peak in the KS potential in the artificial world of KS particles. It is, therefore, not surprising that the universality of the physics in the dissociation limit is reflected in the universal form of the asymptotic KS potential. Since for DFT, only the density distribution is essential the general condition that determines the KS potential in the dissociation limit is simply

$$
n(\mathbf{r})=n_{1}(\mathbf{r})+n_{2}(\mathbf{r}) .
$$

In other words, the total density $n(\mathbf{r})$ is equal to the plain sum of the densities, $n_{1}(\mathbf{r})$ and $n_{2}(\mathbf{r})$, of the two independent fragments. The asymptotic form of the KS potential should be such that it supports the density distribution given by Eq. (18). As the densities $n_{1}(\mathbf{r})$ and $n_{2}(\mathbf{r})$ decay exponentially from different sides the only way to mimic this at the level of a single KS orbital is to insert a potential peak in the middle region. Having understood the key physics we are ready to go to more complex systems.

\section{B. Kohn-Sham potential of heteroatomic 1D molecules}

It is now straightforward to find the form of the KS potential in the dissociation limit for a general 1D molecule formed by two different wells. Assuming that the densities, 
$n_{1}(x)$ and $n_{2}(x)$, corresponding to one electron sitting in a separate well are known, we require that the total density $n(x)$ is given by their sum, Eq. (18), and substitute this sum into Eq. (2). The result can be reduced to a form that looks structurally similar to Eq. (11),

$$
\Delta v_{s}(x)=\frac{\left[\sqrt{n_{1}} \partial_{x} \sqrt{n_{2}}-\sqrt{n_{2}} \partial_{x} \sqrt{n_{1}}\right]^{2}}{2\left(n_{1}+n_{2}\right)^{2}}+\frac{I_{1} n_{1}+I_{2} n_{2}}{n_{1}+n_{2}}-I,
$$

where $I_{1,2}$ are the ionization potentials of the fragments and $I=\min \left\{I_{1}, I_{2}\right\}$ is the ionization potential of the total system. Equation (19) is valid in the dissociation limit, and from its structure it is clear that $\Delta v_{s}(x)$ has a nontrivial $x$-dependence (i.e., differs from a constant) only far away from the "atoms," where the densities fall off exponentially. Therefore, for the practical evaluation of $v_{s}$ in the dissociation limit, it is sufficient to know only the asymptotic behavior of the density of the separate fragments. In the $1 \mathrm{D}$ case, the asymptotics of the densities $n_{1}(x)$ and $n_{2}(x)$ have the following general form

$$
n_{1,2}(x)=A_{1,2} e^{-2 \alpha_{1,2}|x \pm d / 2|},
$$

where the exponents $\alpha_{1,2}$ are related to the ionization potentials of the atoms $I_{1,2}=\alpha_{1,2}^{2} / 2$ and $A_{1,2}$ are prefactors to the exponential decay.

Inserting Eq. (20) for a symmetric molecule (equivalent wells with $A_{1}=A_{2}$, and $\alpha_{1}=\alpha_{2}$ ) into Eq. (19) we immediately recover our first model result of Eq. (12) thus confirming its universality. In a general asymmetric case (different wells or a "heteroatomic" molecule) a new qualitative feature, a "step," appears. This step in $v_{s}$ is such that it aligns the ionization potentials of the atoms. Formally, it results from the last two terms in Eq. (19) which do not cancel if the ionization potentials are different. Substituting the general form of Eq. (20) into Eq. (19) we find the explicit results for $v_{s}$ in different regions of space. It is convenient to represent the KS potential as a sum of two different contributions, i.e., $\Delta v_{s}(x)=v_{s}^{(1)}(x)+v_{s}^{(2)}(x)$. For the region between the wells, i.e., for $-d / 2<x<d / 2$, the two contributions correspond to the "peak" and the "step" discussed before. They are given by

$$
\begin{aligned}
& v_{s}^{(1)}(x)=\frac{\left(\alpha_{1}+\alpha_{2}\right)^{2} / 8}{\cosh ^{2}\left[\left(\alpha_{1}+\alpha_{2}\right)\left(x+x_{0}\right)\right]}, \\
& v_{s}^{(2)}(x)=\frac{I_{2}-I_{1}}{1+\exp \left[2\left(\alpha_{1}+\alpha_{2}\right)\left(x+x_{0}\right)\right]},
\end{aligned}
$$

with

$$
x_{0}=\frac{1}{\alpha_{1}+\alpha_{2}}\left[\frac{\alpha_{1}-\alpha_{2}}{2} \cdot d+\log \frac{A_{2}}{A_{1}}\right] .
$$

Here, and in the following, we assumed that $\alpha_{1} \leq \alpha_{2}$, i.e., that the left fragment has a larger ionization potential. Obviously, for a symmetric configuration, $v_{s}^{(2)}$ vanishes identically, i.e., there is only a peak in this case. Also, in this case $x_{0}=0$, i.e., the peak is exactly in the middle between the two identical fragments as expected from symmetry. The position of the peak and shelf has been derived for a specific model system in Ref. 16 while the result in Eq. (23) is more general.

For $x<-d / 2$ the two contributions read

$$
\begin{aligned}
& v_{s}^{(1)}(x)=\frac{\left(\alpha_{1}-\alpha_{2}\right)^{2} / 8}{\cosh ^{2}\left[\left(\alpha_{1}-\alpha_{2}\right)\left(x+x_{0}^{\prime}\right)\right]}, \\
& v_{s}^{(2)}(x)=\frac{I_{2}-I_{1}}{1+\exp \left[-2\left(\alpha_{1}-\alpha_{2}\right)\left(x+x_{0}^{\prime}\right)\right]},
\end{aligned}
$$

with

$$
x_{0}^{\prime}=\frac{1}{\alpha_{1}-\alpha_{2}}\left[\frac{\alpha_{1}+\alpha_{2}}{2} d-\log \frac{A_{2}}{A_{1}}\right] .
$$

Contrary to before, $v_{s}^{(1)}$ does not describe a peak but it can actually be shown that the potential is strictly monotonically increasing describing the building up of the step, or its return to zero depending on the direction one approaches $x_{0}^{\prime}$ from. Also, for the symmetric case, both contributions vanish as there is no step in that case. For the region $x>d / 2$ the potential decays exponentially without specific features.

We emphasize that neither the specific form of the fragments nor the type of interaction between the electrons enters the derivation of our analytical result directly. The specifics of the fragments appear in the result only via the parameters $\alpha_{1,2}$ and $A_{1,2}$. The former describes how fast the density decays, i.e., it is directly related to the ionization potential of each fragment. The latter is connected to the normalization of the wave function and only enters the potential as a logarithmic correction to the position of the peak and step without changing the shape of the potential. For a symmetric system the potential is completely determined by the ionization potential of the two fragments. In all cases, symmetric and asymmetric, the functional form of the $\mathrm{KS}$ potential is universal; only the position and the width and height of the peak depend on the system under consideration. Both in the symmetric and in the asymmetric case, the presence of the universal peak reflects Hubbard correlations. The potential peak suppresses the tunneling and drives the KS density to the density corresponding to physically independent subsystems.

To ensure that our universal analytical formulas are indeed correct, we performed numerical calculations for an asymmetric two-electron system with the external potential given by the sum of two different potential wells,

$$
v_{\text {ext }}(x)=-\frac{v_{1}}{\cosh ^{2}(x-d / 2)}-\frac{v_{2}}{\cosh ^{2}(x+d / 2)},
$$

with $v_{1}=0.9$ and $v_{2}=0.7$. As before, for the interaction we keep the finite range potential of Eq. (14). Since the oneparticle problem with $1 / \cosh ^{2}$ is exactly solvable, ${ }^{24}$ the parameters $\alpha_{1,2}$ and $A_{1,2}$, entering our asymptotic formulas Eqs. (21)-(26), are available in the analytic form. In particular, for the pre-exponential factors in the asymptotics of the "atomic" densities, we get

$$
A_{1,2}=2^{2 \alpha_{1,2}} \frac{\Gamma\left(\alpha_{1,2}+1 / 2\right)}{\sqrt{\pi} \Gamma\left(\alpha_{1,2}\right)}
$$

where $\Gamma$ denotes the usual gamma-function. 


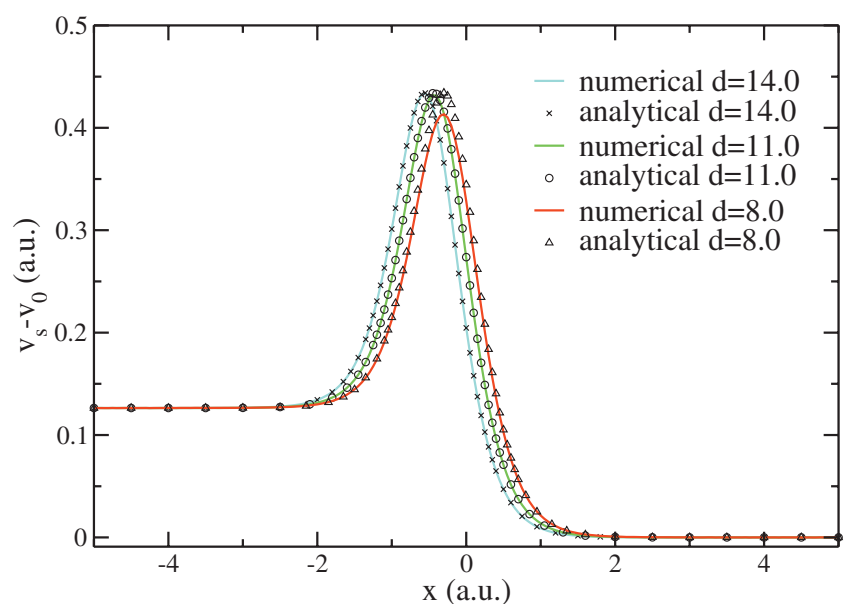

FIG. 2. KS potential for two different wells at different distances $\left(v_{1}=0.9, v_{2}=0.7\right)$. The external potential has been subtracted to facilitate the comparison. Analytical results are given by Eqs. (21) and (22). The potential returns to zero at large negative $x$.

In Fig. 2 we show the comparison of the analytic KS potential given by Eqs. (21) and (22) and the KS potential obtained from the full numerical solution of the problem defined by Eqs. (3), (14), and (27). As expected, in the asymmetric case, $v_{1} \neq v_{2}$, the KS potential acquires a step structure in addition to the peak. The step is a direct result of the necessary alignment of the KS energy levels (the ionization potentials) in the two fragments. ${ }^{9,27}$ It is already not so surprising to see that the KS potential again approaches the analytic asymptotic form with increasing distance. As in the analytic calculation, the exact position of the peak and the step depends slightly on the distance between the two wells always being closer to the deeper well. While in the symmetric case, see Fig. 1, the dissociation limit is reached at a distance of around 8 a.u. in the asymmetric case around 11 a.u. are necessary. In both cases the numerical results agree perfectly with the analytical expression. The larger distance, necessary in the asymmetric case, is a result of the shallower right potential well in that case.

Unfortunately, the analytical result of the step returning to zero cannot be verified numerically for the systems at hand. The position $x_{0}^{\prime}$, Eq. (26), is so far away from the actual potential wells that the density is numerically zero. There is, however, no doubt that the step returns to zero exactly as predicted by the analytic formula.

\section{Generalizations to three-dimensional and many-electron systems}

The general argumentation used in the previous subsection to derive the exact $\mathrm{KS}$ potential in the dissociation limit is not restricted to $1 \mathrm{D}$ systems. The general physical condition for dissociation is that the density is given by the sum of the densities of the independent fragments, Eq. (18), because the interfragment tunneling is destroyed by Coulomb correlations. The inversion formula of Eq. (2) is also valid for any two-particle system independently of dimension. Therefore, an elementary 3D generalization of Eq. (19) takes the form

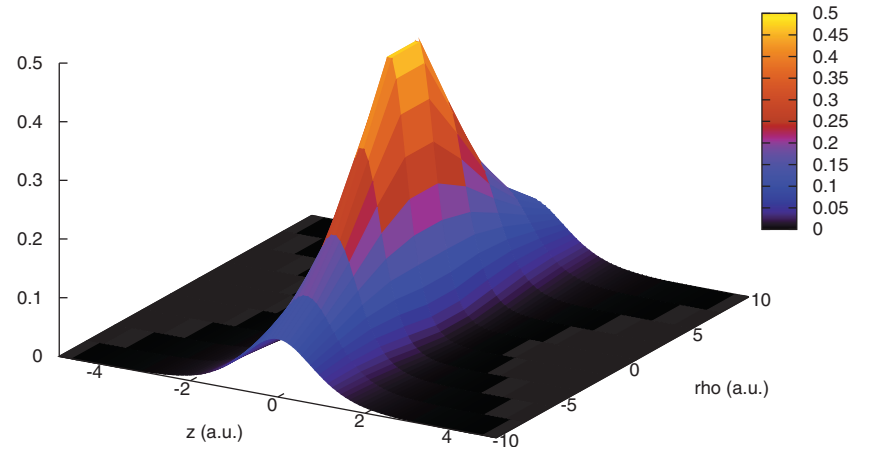

FIG. 3. KS potential of the hydrogen molecule in the dissociation limit.

$$
\Delta v_{s}(\mathbf{r})=\frac{\left[\sqrt{n_{1}} \nabla \sqrt{n_{2}}-\sqrt{n_{2}} \nabla \sqrt{n_{1}}\right]^{2}}{2\left(n_{1}+n_{2}\right)^{2}}+\frac{I_{1} n_{1}+I_{2} n_{2}}{n_{1}+n_{2}}-I,
$$

where all notations are the same as in Eq. (19). Using this formula we can recover the exact limiting functional form of the KS potential for any two-particle object dissociating into two one-particle fragments. The only required input is the long-range asymptotics of the independent fragments, which is mainly determined by their ionization potentials. It is important to emphasize that the pre-exponential factors give only weak logarithmic corrections to the position of the peak and the step.

As an illustration, we present the exact KS potential that controls the dissociation limit of the $\mathrm{H}_{2}$ molecule. The final results obtained by inserting the ionization potential of the hydrogen atom, and the electronic densities of two independent hydrogen atoms, located at the points $\mathbf{R}_{1}$ and $\mathbf{R}_{2}$, into Eq. (29) takes the form

$$
\Delta v_{s}^{\mathrm{H}_{2}}(\mathbf{r})=\frac{1-\mathbf{r}_{1} \mathbf{r}_{2} / r_{1} r_{2}}{4 \cosh ^{2}\left(r_{1}-r_{2}\right)},
$$

where $\mathbf{r}_{1,2}=\mathbf{r}-\mathbf{R}_{1,2}$ are the vectors between the two protons and the considered point in space. The KS potential for the hydrogen molecule, Eq. (30), is shown on Fig. 3. It is easy to see from Eq. (30) that along the molecular axis $\Delta v_{s}^{\mathrm{H}_{2}}(\rho=0, z)$ is exactly of the 1D form Eq. (12), while in the perpendicular direction it has a Lorentzian shape,

$$
\Delta v_{s}^{\mathrm{H}_{2}}(\rho, z=0)=\frac{1}{2} \frac{1}{\left(\frac{2 \rho}{d}\right)^{2}+1},
$$

with the width increasing at increasing distance between the two hydrogen atoms.

Similarly, we can obtain an explicit form of the exact KS potential for any two-electron system in the strongly correlated dissociation limit. Moreover, one can argue that the general formula (29) remains valid also for many electron systems in those cases where the separate fragments have a single electron in the highest occupied KS orbital. Indeed, in this case the asymptotic behavior of the density away from the atoms is completely determined by the two KS particles in the highest occupied KS molecular orbital (KS HOMO), while the rest of the electrons effectively contribute to the 
rigid atomic cores. Therefore, the asymptotic form of the KS potential can be obtained by inverting only one KS equation, namely, for the KS HOMO, and, hence, the two-particle formula (2) remains asymptotically valid. In the general case, $\Delta v_{s}$ in Eq. (29) describes the difference between the actual $v_{s}$ and the sum of the KS potentials of the two independent fragments.

\section{CONCLUSIONS}

In conclusion, we presented a recipe to calculate the exact KS potential of systems in their dissociation limit. The main ingredient is the ionization potential of the dissociated fragments, a quantity that is readily available from spectroscopic data. We presented the explicit results for a 1D model system and the hydrogen molecule. It is shown that the functional form of the potential is independent of the specific system and the details of the interaction as long as the latter is repulsive and sufficiently strong. For the 1D model system the numerical results approach the analytical one as the distance between the two fragments is increased. Hence, they confirm our analytical result perfectly for both a symmetric and an asymmetric system. Our results not only pose a strong constraint for the development of xc functionals but also introduce an alternative way to look at the electron localization in strongly correlated systems. How to incorporate those effects in a density-functional treatment remains a challenge. It is especially intriguing to explore implications of our universal results for the quantum transport in the regime of Coulomb blockade. It is natural to expect that the potential peak in the KS potential should modify the tunneling probability when the transport is described in terms of KS DFT.

\section{ACKNOWLEDGMENTS}

We acknowledge funding by the Spanish MEC (Grant No. FIS2007-65702-C02-01), "Grupos Consolidados UPV/ EHU del Gobierno Vasco" (Grant No. IT-319-07), and the European Community through e-I3 ETSF project (Grant Agreement No. 211956).

\section{APPENDIX: CONNECTION TO THE KINETIC- CORRELATION POTENTIAL}

In this appendix we make a connection of our results to the partitioning of the KS potential introduced in Ref. 10, and to the interpretation of the peak in $v_{s}$ in terms of the conditional probability. ${ }^{14}$ The key object is an amplitude of the conditional probability that, for a general $N$-particle system, is defined as follows:

$$
\Phi\left(\mathbf{r}_{2}, \ldots, \mathbf{r}_{N} \mid \mathbf{r}_{1}\right)=\frac{\Psi\left(\mathbf{r}_{1}, \mathbf{r}_{2}, \ldots, \mathbf{r}_{N}\right)}{\sqrt{n\left(\mathbf{r}_{1}\right) / N}},
$$

where $\Psi\left(\mathbf{r}_{1}, \mathbf{r}_{2}, \ldots, \mathbf{r}_{N}\right)$ is the ground-state many-body wave function. The square of the function $\Phi\left(\mathbf{r}_{2}, \ldots, \mathbf{r}_{N} \mid \mathbf{r}_{1}\right)$, Eq. (A1) gives a probability distribution for $N-1$ particles when the position of a selected "reference" particle is fixed at $\mathbf{r}_{1}$.
In Refs. 10 and 14 the peak structure in $v_{s}$ has been attributed to a "kinetic-correlation" part, $v_{\text {kin }}$, of the decomposition of the KS potential proposed in Ref. 10. In terms of the conditional amplitude it is given as

$$
v_{\text {kin }}\left(\mathbf{r}_{1}\right)=\frac{1}{2} \int\left|\nabla_{\mathbf{r}_{1}} \Phi\left(\mathbf{r}_{2}, \ldots, \mathbf{r}_{N} \mid \mathbf{r}_{1}\right)\right|^{2} d \mathbf{r}_{2} \ldots d \mathbf{r}_{N}
$$

Physically, $v_{\text {kin }}(\mathbf{r})$ can be interpreted as an "integrated rate" of change of the conditional probability under an infinitesimal shift of the reference electron from a given point $\mathbf{r}$. Hence, a peak in $v_{\text {kin }}(\mathbf{r})$ in the dissociation limit signals that the conditional probability changes significantly when the reference electron crosses over the middle point between two fragments. To make the connection of this interpretation to our universal results as clear and explicit as possible, we employ the analytically solvable model with an infinite delta repulsion, which has been discussed in Sec. II B.

The exact ground state wave function is given by Eq. (6). Inserting this wave function into Eqs. (A1) and (A2) we obtain for the correlation-kinetic potential

$$
\begin{aligned}
v_{\text {kin }}(x) & =\frac{1}{2}\left(\frac{d}{d x} \frac{\phi_{+}(x)}{\sqrt{n(x)}}\right)+\frac{1}{2}\left(\frac{d}{d x} \frac{\phi_{-}(x)}{\sqrt{n(x)}}\right) \\
& =\frac{\left(\phi_{+} \phi_{-}^{\prime}-\phi_{+}^{\prime} \phi_{-}\right)^{2}}{2\left(\phi_{+}^{2}+\phi_{-}^{2}\right)^{2}},
\end{aligned}
$$

where $\phi_{ \pm}(x)$ are defined after Eq. (8). Comparing the result of Eq. (A3) with the exact KS potential (11) we find that the first term in Eq. (11) is exactly the correlation-kinetic potential. According to our analysis, in the dissociation limit, only this term survives and yields the universal peak, Eq. (12), in the KS potential. Hence, in agreement with Refs. 10 and 14, $v_{\text {kin }}$ is indeed responsible for the peak structure. Apparently, this conclusion is universal and applies to all generalizations considered in Sec. III.

A peak in $v_{\text {kin }}(x)$ should correspond to rapid variations in the conditional probability when the reference particle moves across the middle point in the bonding region. ${ }^{11-13}$ Therefore, it is instructive to look at the behavior of the function $\Phi^{2}\left(x_{2} \mid x_{1}\right)$ for our analytic model,

$$
\Phi^{2}\left(x_{2} \mid x_{1}\right)=\frac{\left[\phi_{+}\left(x_{1}\right) \phi_{-}\left(x_{2}\right)-\phi_{-}\left(x_{1}\right) \phi_{+}\left(x_{2}\right)\right]^{2}}{\phi_{+}^{2}\left(x_{1}\right)+\phi_{-}^{2}\left(x_{1}\right)} .
$$

In the dissociation limit $v d \rightarrow \infty$ this equation can be represented in the following asymptotic form:

$$
\begin{aligned}
\Phi^{2}\left(x_{2} \mid x_{1}\right) \approx & \frac{1-\tanh 2 v x_{1}}{2} v e^{-2 v\left|x_{2}-d / 2\right|} \\
& +\frac{1+\tanh 2 v x_{1}}{2} v e^{-2 v\left|x_{2}+d / 2\right|} .
\end{aligned}
$$

Equation (A5) clearly shows how the strong Hubbard correlations, which are present in the ground-state wave function, govern the behavior of the conditional probability of the second particle when the reference particle is moving from the left to the right across the system. When the reference electron is on the left hand side, at $x_{1}<-1 / 2 v$, the first term in Eq. (A5) dominates and the second particle is forced to sit on 
the right atom. If the reference particle is located on the right hand of the system, at $x_{1}>1 / 2 v$, the situation is oppositethe second term in Eq. (A5) dominates and the second particle is repelled to the left atom. The crossover between these two regimes occurs when the reference electron crosses the middle region, in particular at $x_{1}=0$ the probability to find the second particle on either atom is equal to 1/2. Exactly this crossover behavior is reflected in the peak of $v_{\text {kin }}(x)$ and, therefore, in the KS potential

${ }^{1}$ P. Hohenberg and W. Kohn, Phys. Rev. 136, B864 (1964).

${ }^{2}$ W. Kohn and L. J. Sham, Phys. Rev. 140, A1133 (1965).

${ }^{3}$ M. A. L. Marques and A. Rubio, Phys. Chem. Chem. Phys. 11, 4421 (2009).

${ }^{4}$ M. Casida, H. Chermette, and D. Jacquemin, J. Mol. Struct.: THEOCHEM 914, 1 (2009).

${ }^{5}$ M. Fuchs, Y.-M. Niquet, X. Gonze, and K. Burke, J. Chem. Phys. 122, 094116 (2005).

${ }^{6}$ J. Dobson, in Time-Dependent Density Functional Theory (Springer, Berlin, Heidelberg, 2006), pp. 443-462.

${ }^{7}$ V. I. Anisimov, F. Aryasetiawan, and A. I. Lichtenstein, J. Phys. C 9, 767 (1997).

${ }^{8}$ A. Georges, G. Kotliar, W. Krauth and M. Rozenberg, Rev. Mod. Phys. 68, 13 (1996); G. Kotliar and D. Vollhardt, Physics Today 57, 53 (2004); G. Kotliar, S. Y. Savrasov, K. Haule, V. S. Oudovenko, O. Parcollet, and C. A. Marianetti, Rev. Mod. Phys. 78, 865 (2006).

${ }^{9}$ C. O. Almbladh and U. von Barth, Density Functional Methods in Physics (Plenum, New York, 1985), pp. 209-231; J. P. Perdew, Density Functional Methods in Physics (Plenum, New York, 1985), pp. 265-308.
${ }^{10}$ M. A. Buijse, E. J. Baerends, and J. G. Snijders, Phys. Rev. A 40, 4190 (1989).

${ }^{11}$ R. van Leeuwen and E. J. Baerends, Phys. Rev. A 49, 2421 (1994).

${ }^{12}$ O. V. Gritsenko, R. van Leeuwen, and E. J. Baerends, Phys. Rev. A 52, $1870(1995)$

${ }^{13}$ O. V. Gritsenko and E. J. Baerends, Phys. Rev. A 54, 1957 (1996).

${ }^{14}$ O. V. Gritsenko, R. van Leeuwen, and E. J. Baerends, J. Chem. Phys. 104, 8535 (1996).

${ }^{15}$ O. V. Gritsenko and E. J. Baerends, Theor. Chem. Acc. 96, 44 (1997).

${ }^{16}$ D. G. Tempel, T. J. Martínez, and N. T. Maitra, J. Chem. Theory Comput. 5, 770 (2009).

${ }^{17}$ Apparently, the KS potential close to each of the dissociated fragments is identical to the potential of the isolated fragment. When we are talking about universality we are referring to the nontrivial features of the KS potential between the fragments.

${ }^{18}$ A. Becke, Phys. Rev. A 38, 3098 (1988).

${ }^{19}$ J. P. Perdew, Phys. Rev. B 33, 8822 (1986).

${ }^{20}$ R. van Leeuwen and E. J. Baerends, Int. J. Quantum Chem. 52, 711 (1994).

${ }^{21}$ M. Girardeau, J. Math. Phys. 1, 516 (1960).

${ }^{22}$ V. I. Yukalov and M. D. Girardeau, Laser Phys. Lett. 2, 375 (2005).

${ }^{23}$ We only consider distances $d>1 / v$ where both lowest states are bound.

${ }^{24}$ L. D. Landau and E. M. Lifschitz, Quantum Mechanics (ButterworthHeinemann, Oxford, 1977).

${ }^{25}$ M. A. L. Marques, A. Castro, G. F. Bertsch, and A. Rubio, Comput. Phys. Commun. 151, 60 (2003); A. Castro, H. Appel, M. Oliveira, C. A. Rozzi, X. Andrade, F. Lorenzen, M. A. L. Marques, E. K. U. Gross, and A. Rubio, Phys. Status Solidi B 243, 2465 (2006).

${ }^{26}$ We note that in the case of a long-range soft Coulomb interaction we encountered numerical instabilities at too large distances.

${ }^{27}$ N. Maitra, J. Chem. Phys. 122, 234104 (2005). 